\title{
Effect of screening on deaths from cervical cancer in Sweden
}

In the online version of this Editorial by M Arbyn and colleagues (BMJ 2012;344:e804, doi:10.1136/bmj.e804) incidence and

Cite this as: BMJ 2012;344:e1705

mortality are the wrong way round in the figure. The red dashed

๑ BMJ Publishing Group Ltd 2012

line should be incidence and the continuous line should be

mortality. 